\title{
Chain-Growth Condensation Polymerization of Propargyl Electrophiles Enabled by Copper Catalysis
}

Han-Li Sun, ${ }^{1}$ Da-Qi Liu, ${ }^{2,3}$ Jun-Jie Wang, ${ }^{1}$ Dawen $\mathrm{Niu}^{2,3}$ and Rong Zhu ${ }^{1 *}$

${ }^{1}$ Beijing National Laboratory for Molecular Sciences, Key Laboratory of Bioorganic Chemistry and Molecular Engineering of Ministry of Education, College of Chemistry and Molecular Engineering, Peking University, Beijing 100871, China

${ }^{2}$ Department of Emergency, State Key Laboratory of Biotherapy, West China Hospital, Sichuan University, Chengdu, China

${ }^{3}$ School of Chemical Engineering, Sichuan University, Chengdu, China

*e-mail: rongzhu@pku.edu.cn (R. Zhu) 


\begin{abstract}
In the pursuit of creating macromolecules with controlled molecular weight, sequence, and end groups, condensation polymerization remains an underexploited synthetic tool because of its intrinsic step-growth nature. Introducing chain-growth pathways into condensation polymerization calls for highly efficient chemistries that effect the challenging differentiation between functional groups of the same type present in monomers and polymers. Here, we address this challenge by a catalyst bifurcation strategy that enables a copper-catalyzed chain-growth condensation polymerization. Using a copper(I) arylacetylide as an initiator/precatalyst along with a phosphine ligand, polydiynes of controllable molecular weights and end groups are synthesized from readily available propargyl carbonates, including a block copolymer. This method provides a new chaingrowth access to functional acetylenic polymers, a class of useful materials that have been obtained essentially by step-growth methods to date. This work demonstrates the power of dual-role transition metal catalysis in accomplishing unusual selectivity in organic synthesis.
\end{abstract}




\section{Introduction}

The precise control of the molecular weight (MW), sequence, and end groups of a synthetic polymer has been best demonstrated in living chain-growth polymerizations involving addition, ring-opening, and group transfer processes. ${ }^{1}$ In contrast, achieving such control in conventional condensation polymerizations has been elusive due to their intrinsic step-growth nature, which limits the engineering of the properties of the resulting materials. To address this issue, several elegant strategies have recently been developed to enable chain-growth condensation polymerizations (CGCP) of certain bifunctional monomers (Figure 1a). ${ }^{2}$ The key to CGCP is to differentiate between the same type of functional groups present at a polymer chain end and in a monomer, respectively. For example, CGCP accesses to well-defined polyamides, polyesters, and polyethers were developed by Yokozawa based on substituent effect or phase transfer catalysis. ${ }^{3}$ Catalyst transfer, pioneered by Yokozawa and McCullough, represents another prominent example, which allows for controlled synthesis of conjugated polymers. ${ }^{4}$ Additionally, CGCP via fluoride transfer, $\mathrm{S}_{\mathrm{RN}} 1$ and cationic mechanisms have been reported. ${ }^{5}$ Despite these advances, the scope of CGCP is still quite limited, arguably due to the difficulty in identifying chemistries for effective reactivity differentiation.

As part of the ongoing program exploring dual-role catalysis involving first-row transition metals, we became interested in leveraging such systems for new CGCP reactions (Figure 1b). ${ }^{6}$ We envisioned that metal catalysts could activate a polymer chain end and a monomer in parallel, which might give a pair of organometallic intermediates that display strong nucleophilicity and electrophilicity, respectively. As a result, the cross-condensation between these activated species would be facile, thereby accomplishing chain-growth by leaving one of the metal centers at the 
terminal. In contrast, the self-condensation between the activated monomers would be much slower due to the mismatched polarity.

Specifically, we targeted propargyl electrophiles, for instance, a propargyl carbonate $\mathbf{M}$ (Figure 1c). ${ }^{7}$ It has been suggested that in $\mathbf{C u}$-catalyzed propargylic substitution reactions, $\mathbf{M}$ is activated via a copper acetylide A, which is en route to a putative electrophilic copper allenylidene species $\mathbf{B}$. ${ }^{6 c, 8}$ In the context of polymerization, we hypothesized that $\mathbf{B}$, at either the $\alpha$-or $\gamma$-position, could be selectively intercepted by a chain-carrying copper species $\mathbf{C}$ rather than by $\mathbf{A}$, which is deactivated due to the electron-withdrawing hyperconjugation posed by the carbonate leaving group. This reactivity differentiation would eventually lead to a $\mathrm{Cu}$-catalyzed chain-growth polycondensation of propargyl electrophiles (Cu-Catalyzed CPPE). Here we report the materialization of this proposal, which furnishes polydiynes in a controlled chain-growth fashion that is distinct from conventional step-growth approaches such as the Glaser-Hay oxidative coupling (Figure 1d). ${ }^{9}$ 
a)

- Electrophilic Site Deactivation in Monomer

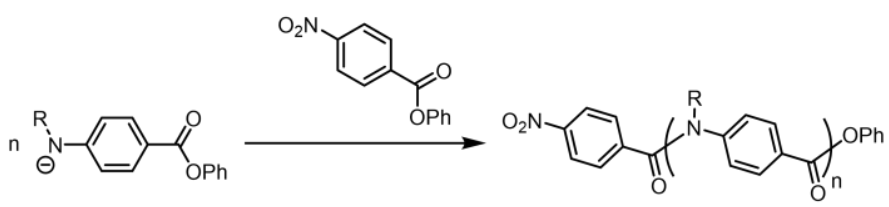

- Catalyst Transfer
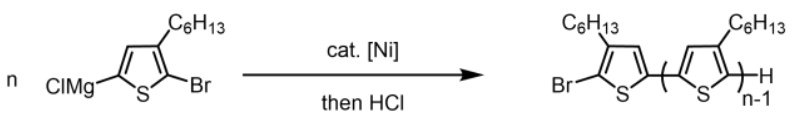

b)

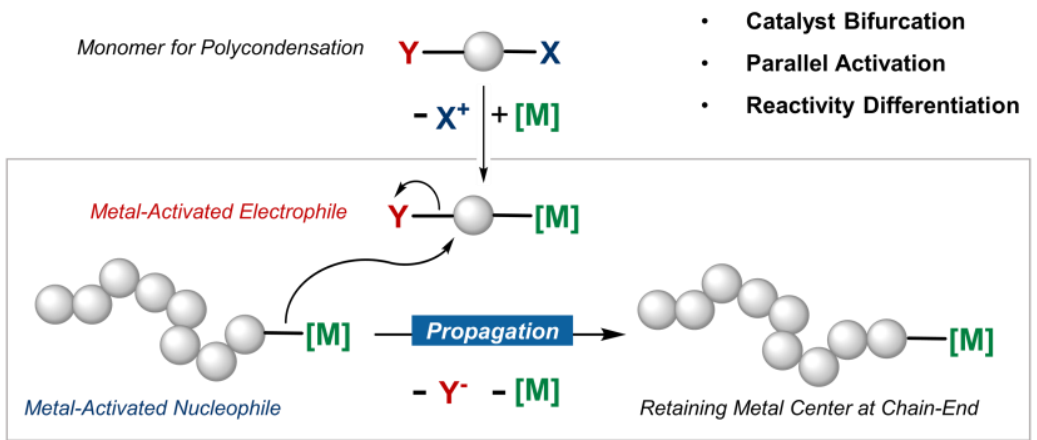

c)

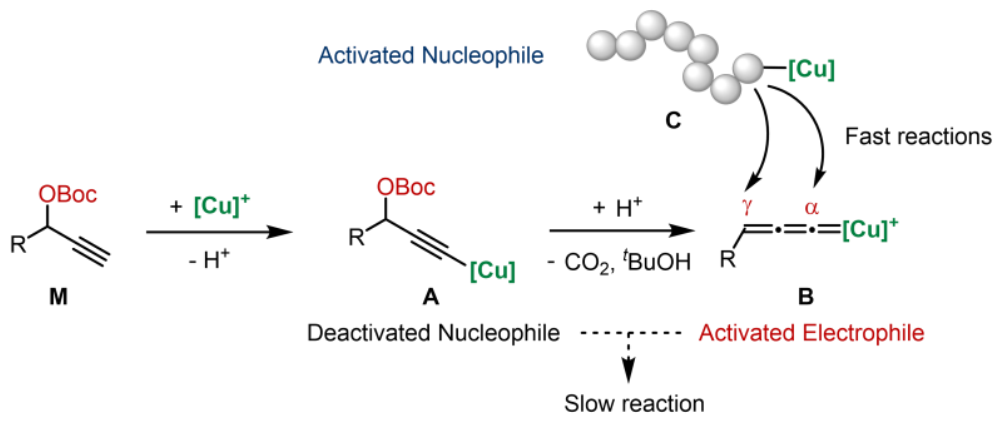

d)

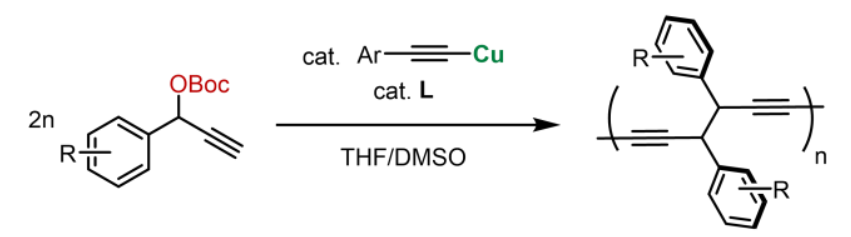

- Chain-Growth - Controllable MW/End Group - Access to Block Copolymers

Figure 1. a) Representative strategies for chain-growth condensation polymerizations (CGCP). b)

Proposed new CGCP via transition metal enabled parallel activation. c) Possible reactivity differentiation in $\mathrm{Cu}$-catalyzed propargylic substitution reactions. d) This work: $\mathrm{Cu}$-catalyzed chaingrowth polycondensation of propargyl electrophiles (CPPE). 


\section{Result and Discussion}

Reaction development. For a preliminary test, we studied the reaction of propargyl carbonate M1 in the presence of catalytic amounts of $\mathrm{Cu}(\mathrm{MeCN})_{4} \mathrm{PF}_{6}$ and triethylamine under nitrogen atmosphere (Scheme 1, eq. 1). By itself, M1 dimerizes regioselectively in an $\alpha-\alpha$ fashion, presumably via an organocopper species $\mathbf{D}$, to afford an allene-yne $\mathbf{1}$ and a diyne $\mathbf{1}^{\prime} .{ }^{10}$ It was noticed that $\mathbf{1}$ was unstable and gradually isomerized to $\mathbf{1}^{\prime}$. In the presence of phenyl acetylene, however, the formation of crosscondensation adducts $\mathbf{2} / \mathbf{2}^{\prime}$ (> 20\%) was predominant over self-condensation (3\%) (eq. 2). ${ }^{11}$ This observed preference for cross-condensation strongly supports the hypothesized reactivity differentiation. The addition of a bulky biaryl dialkylphosphine ligand XPhos (L1) was found to further boost the selectivity ( $>38: 1) .{ }^{12}$ Importantly, Glaser coupling products were not detected under these conditions. ${ }^{13}$ 


\section{Scheme 1. Small molecule model reactions.}
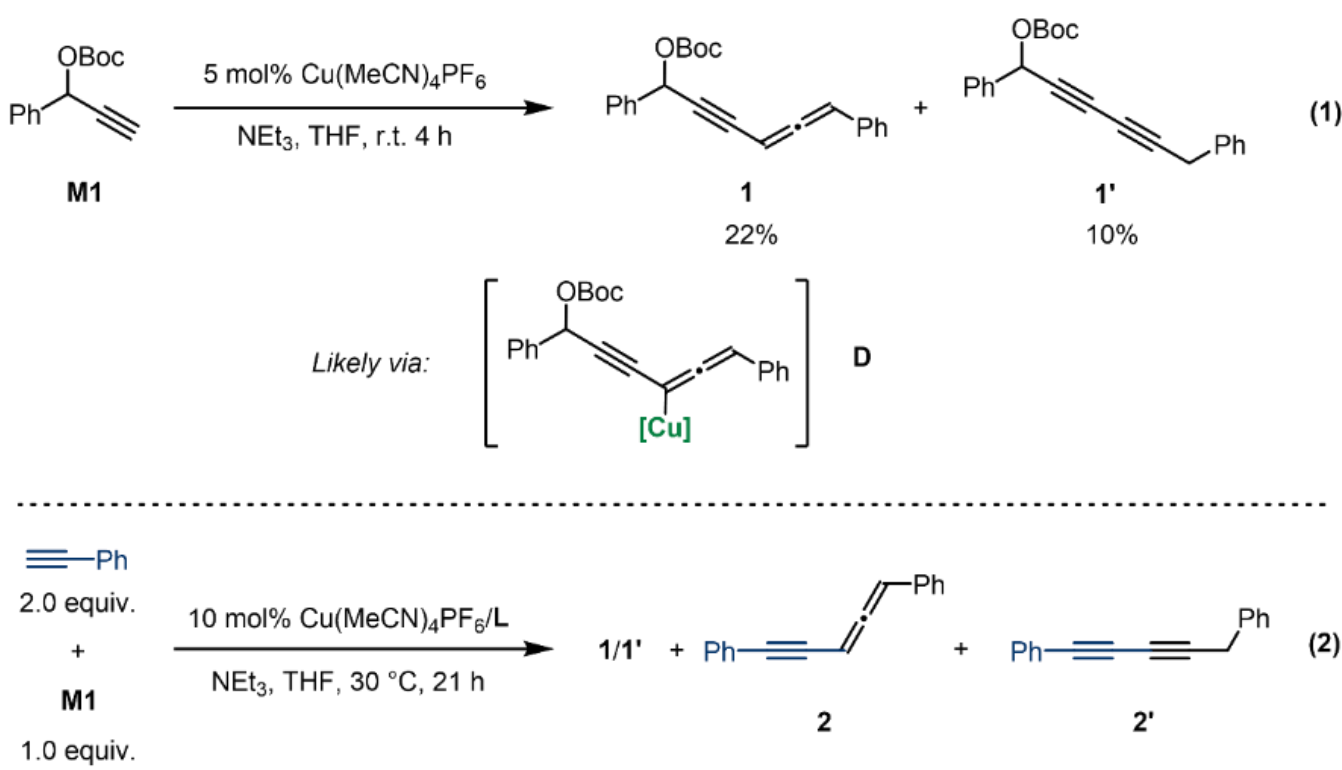

\begin{tabular}{|c|c|c|c|}
\hline $\mathbf{L}$ & $1+1^{\prime}$ & 2 & \\
\hline None & $3 \%$ & $20 \%$ & \\
\hline L1 & $<1 \%$ & $38 \%$ & L1 XPhos \\
\hline
\end{tabular}

Having noticed that the in situ formed D could serve as a viable initiator because of its nucleophilicity, we first began to investigate the polymerization of M1 under conditions that were similar to those depicted in Scheme 1 (Table 1). In the absence of a ligand, full conversion of M1 was accompanied by the formation of dimers $2 / \mathbf{2}^{\prime}$ along with some higher oligomers, likely indicating a step-growth behavior (entry 1). Guided by the results from small molecule reactions, L1 was employed as a ligand for $\mathrm{Cu}(\mathrm{I})$. Indeed, it provided a significant increase in yield and $\mathrm{MW}$, despite a bimodal MW distribution found in the corresponding gel permeation chromatography (GPC) trace, which might be attributable to a mixed mechanism for initiation and/or growth (entry 2). Bidentate $N$ - and $P$-based ligands such as $2,2^{\prime}$-bipyridyl (L2) and $(R)$-BINAP (L3) were found inferior, and a base proved necessary for initiating the polymerization (entries 3-5).

To achieve a controlled chain-growth polymerization, we turned to highly air- and moisture-stable 
crystalline $\mathrm{Cu}(\mathrm{I})$ acetylides 3 as well-defined and easy-to-handle initiators. ${ }^{14}$ Meanwhile, it was anticipated that a portion of such a complex could play a second role as a basic precatalyst for monomer activation through $\mathrm{Cu}(\mathrm{I}) / \mathrm{H}^{+}$exchange. Switching to $\mathrm{Cu}(\mathrm{I})$ phenylacetylide (3a) as an initiator/precatalyst, M1 was polymerized smoothly till full conversion, which yielded a high MW polymer with a unimodal distribution (entry 6). A narrower dispersity was obtained upon the addition of L1, along with DMSO as a co-solvent to facilitate the dissolution of 3a (entry 7). Dilution and elevated temperature led to increased MW (up to $33.8 \mathrm{~kg} / \mathrm{mol}$ ) but meanwhile broader dispersity (entries 8-10).

As expected, higher loading of 3a led to a decrease in MW (entry 11). When L1 was used in excess compared to 3a, the monomer conversion slowed down but a larger MW was obtained (entries 12 and 13). In the latter case, a relatively low dispersity of 1.6 was noticed. Finally, $\mathrm{Cu}(\mathrm{I}) 4-$ isopropylphenylacetylide (3b) was employed and produced substantially larger polymers than that was obtained using the same loading of 3a (entry 14 vs. 7, 20.9 vs. $7.0 \mathrm{~kg} / \mathrm{mol}$ ). Such complex behaviors might be attributable to that the distribution of the total $\mathrm{Cu}(\mathrm{I})$ between the chain-carrying species and the activated monomers is dependent on the ligand loading as well as the nature of $\mathbf{3}$. Despite of this complication, a reasonable MW control could still be achieved by varying the loading of $\mathbf{3 b}$ (Figure 2a). This mechanistic complexity was also reflected by its kinetic profile (Figure 2b). After an induction period, a rapid propagation seemed to take place initially, producing polymers of $\sim 8 \mathrm{~kg} / \mathrm{mol}$ at $c a .20 \% \mathbf{M 1}$ conversion. It then entered a more controlled regime where the MW increased with monomer conversion, with the dispersity being relatively constant. Collectively, these observations are consistent with a chain-growth character. 
Table 1. Selected polymerization results of M1. ${ }^{a}$

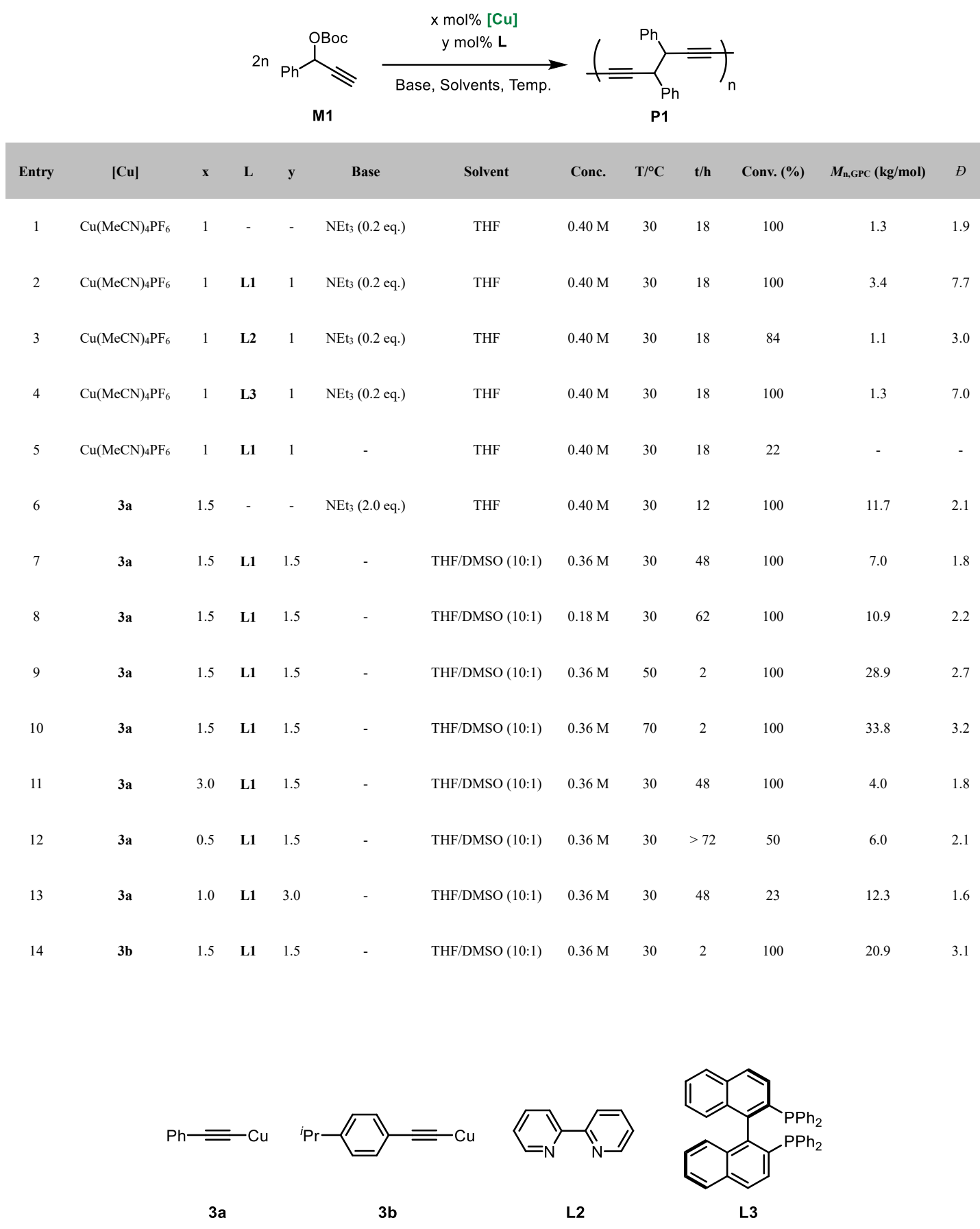

${ }^{a}$ All reactions were carried out using $0.40 \mathrm{mmol}$ of $\mathbf{M 1}$. The monomer conversions were determined by ${ }^{1} \mathrm{H}$ NMR analysis of the crude reaction mixtures. The polymers were isolated by precipitation in hexanes or $\mathrm{MeOH} / \mathrm{H}_{2} \mathrm{O}$. The number-average MW $\left(M_{\mathrm{n}}\right)$ and dispersity $(\nexists)$ were determined by GPC (THF) relative to polystyrene standards. 
a)

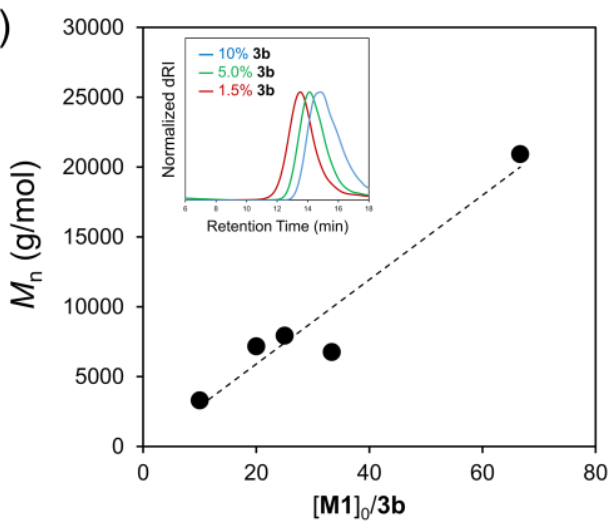

b)

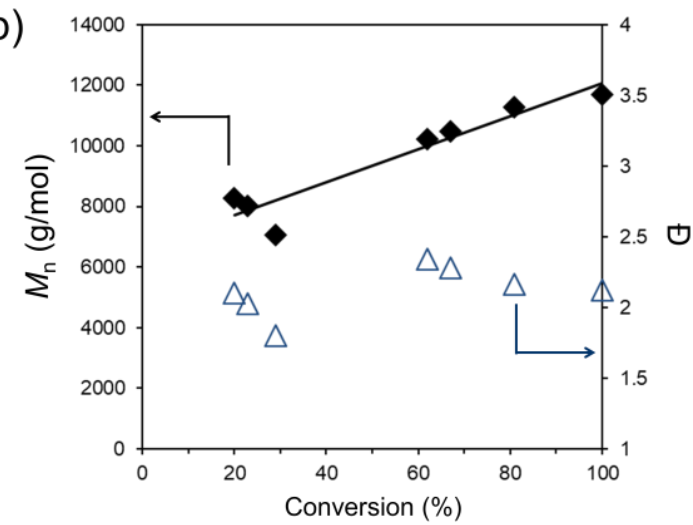

Figure 2. Evaluations of the chain-growth character. a) MW control by varying initiator loadings. Inset: Normalized GPC (THF) traces overlay at different $3 \mathbf{b}$ loadings. Conditions: M1(0.40 mmol), 3b (1.5 - $10 \mathrm{~mol} \%), \mathbf{L 1}(1.5 \mathrm{~mol} \%), \operatorname{THF}(1.0 \mathrm{~mL})$, DMSO $(0.1 \mathrm{~mL}), 30{ }^{\circ} \mathrm{C}$ for 2 h. b) $M_{\mathrm{n}}(\diamond)$ and $Ð(\triangle)$ as functions of monomer conversion. Conditions: M1 $(0.40 \mathrm{mmol}), 3 \mathrm{a}(1.5 \mathrm{~mol} \%)$, triethylamine $(0.80 \mathrm{mmol})$ in $\mathrm{THF}(1.0 \mathrm{~mL})$ at $30^{\circ} \mathrm{C}$.

Characterizations. A series of characterizations were next performed to establish the structure of the polymer (Figure 3). The ${ }^{1} \mathrm{H}$ and ${ }^{13} \mathrm{C}$ NMR spectra of a sample of $\mathbf{P 1}\left(M_{\mathrm{n}, \mathrm{GPC}}=7.2 \mathrm{~kg} / \mathrm{mol}\right.$, initiator $=\mathbf{3 b}$ ) displayed characteristic signals for the benzylic protons/carbons and the 1,3-diyne carbons on the backbone. This suggests that the repeating units were connected exclusively via $\alpha$ $\alpha / \gamma-\gamma$ couplings, and the other possible regiochemistry $(\alpha-\gamma)$ was not detected. The weak diyne $C \equiv$ C bond stretching band $\left(2246 \mathrm{~cm}^{-1}\right)$ was also found in the infrared spectrum (Figure S14). P1 underwent an exothermic process at $c a .160{ }^{\circ} \mathrm{C}$ during thermal analysis, which is attributable to the thermal cross-linking reaction that is characteristic of 1,3-diynes (Figure S18).${ }^{15}$ There was no glass transition observed for $\mathbf{P 1}$ below that temperature, which reflects the rigidity of its backbone. Despite of insulating $s p^{3}$ carbon units in the backbone, the solutions and thin films of $\mathbf{P 1}$ was shown 
to absorb in the blue region and emit at around $500 \mathrm{~nm}$ (Figure S15). At this stage we are in the process of determining the origin of this emission. ${ }^{16}$
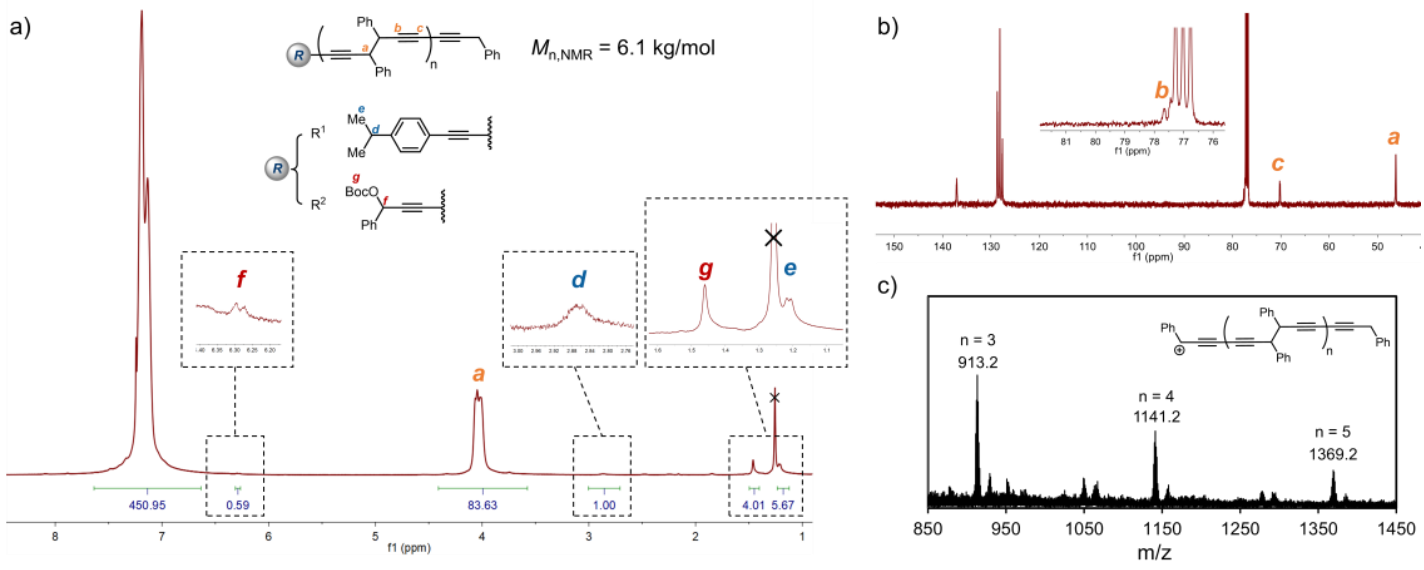

Figure 3. Characterizations of P1. a) b) ${ }^{1} \mathrm{H}$ and ${ }^{13} \mathrm{C}$ NMR spectra $\left(M_{\mathrm{n}, \mathrm{GPC}}=7.2 \mathrm{~kg} / \mathrm{mol}\right.$, initiator $=$ 3b). c) MALDI-TOF mass spectrum $\left(M_{\mathrm{n}, \mathrm{GPC}}=4.0 \mathrm{~kg} / \mathrm{mol}\right.$, initiator $\left.=\mathbf{3 a}\right)$.

A closer look at the ${ }^{1} \mathrm{H}$ NMR spectrum of $\mathbf{P} 1$ revealed the existence of two types of end groups. The major one was derived from initiator $\mathbf{3 b}$ as indicated by the isopropyl group. Meanwhile, a minor end group derived from M1 was observed, implying a competing self-initiation process involving D. The presence of both pathways could be accountable for broadening the MW distribution. An estimation of the MW was thus made based on NMR analysis to be $6.1 \mathrm{~kg} / \mathrm{mol}$, which is reasonably close to the result from GPC measurement. In order to confirm the structure assignment, matrixassisted laser desorption/ionization time-of-flight (MALDI-TOF) mass spectroscopy was attempted on multiple samples of $\mathbf{P 1}$ synthesized under different conditions. However, well-resolved peaks could only be observed in the low $\mathrm{m} / \mathrm{z}$ region corresponding to the self-initiated chains, which contain $-\mathrm{OBoc}$ groups that likely facilitates ionization. Nonetheless, repeating unit of $\mathrm{m} / \mathrm{z}=228$ as well as the end-capping monomer could clearly identified. 
Monomer scope. With the polymerization protocol in hand, we set out to evaluate the $\mathrm{Cu}$-Catalyzed CPPE of a range of aryl- and heteroaryl-bearing propargyl carbonates (Scheme 2). Para- and orthoaryl halides (M2-4) were well-tolerated and the corresponding polymers were obtained in high yields. Such halide groups could potentially provide useful handles for post-polymerization functionalizations. It is noteworthy that for $\mathbf{P} 2$ and $\mathbf{P 3}$, characteristic signals for terminal alkynes were detected in the ${ }^{1} \mathrm{H}$ NMR spectra (S26), which might be attributable to the end-capping protonation of an alternative acetylide chain-end (see mechanistic discussions vide infra). Monomers containing a thiophene (M5) or a naphthalene (M6) group were also successfully employed, which produced polymers of limited solubility. In addition, polymers with an alkoxy (M7) and a TEG (M8) substituents incorporated on the phenyl moiety could be synthesized, albeit in diminished MW and yields. The polymerization of $\mathbf{M 9}$, which has an electron-withdrawing trifluoromethyl group, was unsuccessful as a result of the rapid isomerization to allene 4 . 
Scheme 2. Monomer Scope for Cu-Catalyzed CPPE. ${ }^{a}$

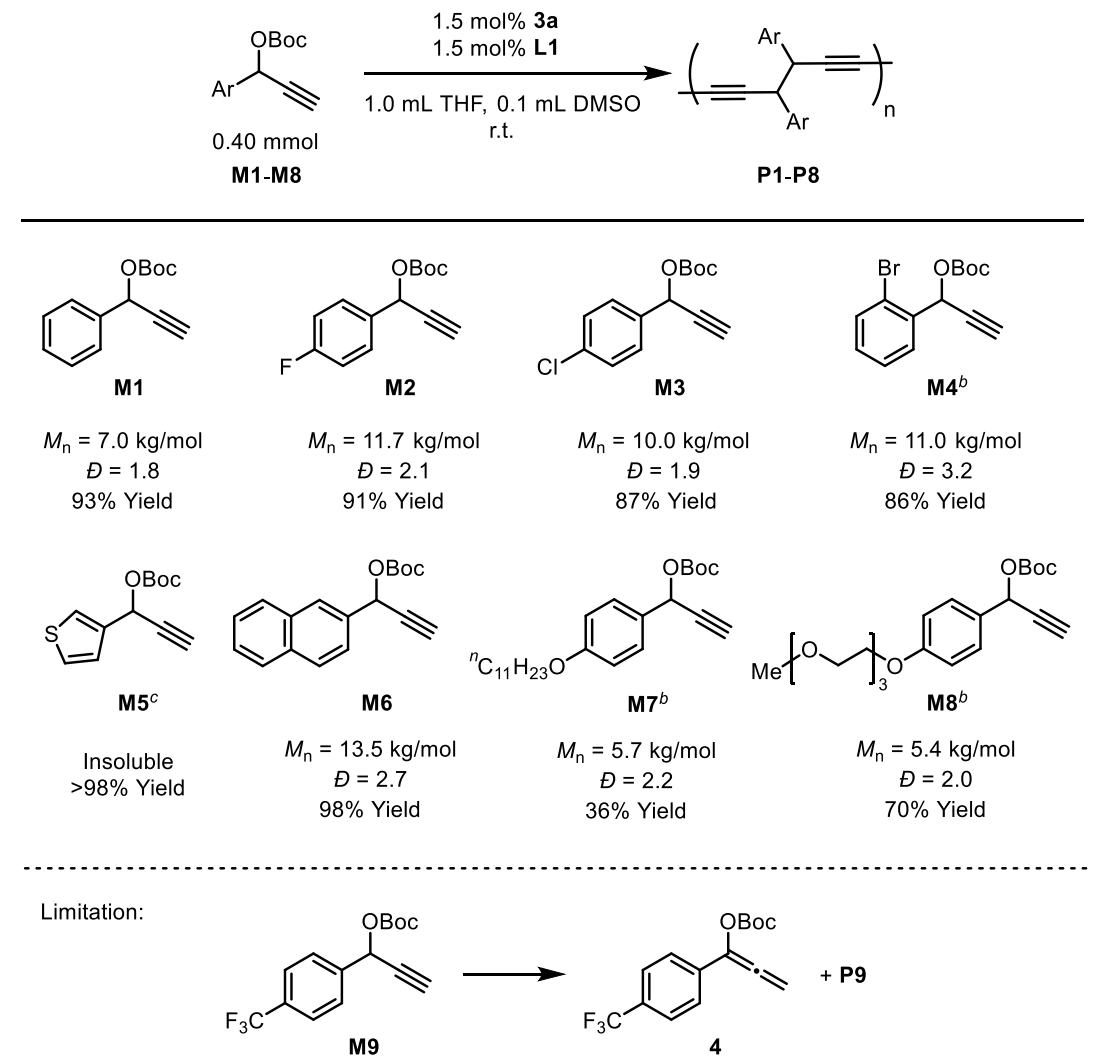

${ }^{a}$ All reactions reached full conversion of the monomer. P1-P8 were isolated by precipitation in hexanes or $\mathrm{MeOH} / \mathrm{H}_{2} \mathrm{O} .{ }^{b}$ At $70{ }^{\circ} \mathrm{C} .{ }^{c}$ Carried out using 3a $(1.5 \mathrm{~mol} \%)$ and $\mathrm{Et}_{3} \mathrm{~N}$ (2.0 equiv.) in THF $(1.0 \mathrm{~mL})$ at $30^{\circ} \mathrm{C}$

Synthesis of a block copolymer. In principle, CGCP should inherently display "living" character, because the reacting sites are stable functional groups unlike radicals or cations. ${ }^{2 a}$ To exploit such character in Cu-catalyzed CPPE, we performed a chain extension experiment to synthesize a block copolymer (Figure 4). 3a-initiated CPPE of M1 was carried out to synthesize the first block. Upon full conversion of M1, a portion of the reaction mixture was taken out and analyzed. A solution of M2 was next added to afford a block copolymer P10 in 87\% yield. As shown in Figure 4, the GPC 
trace of P10 was unimodal and shifted to higher MW compared to that of the first block. The incorporation of $\mathbf{M 2}$ was also confirmed by ${ }^{1} \mathrm{H}$ NMR spectroscopic analysis (Figure S10).
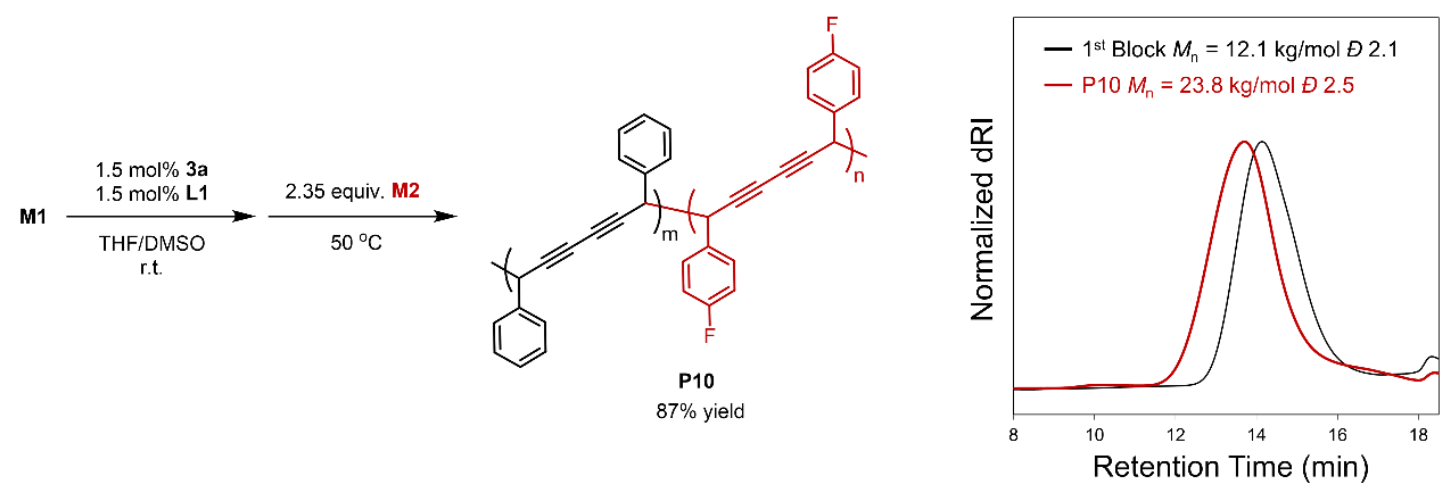

Figure 4. Synthesis of a block copolymer by Cu-catalyzed CPPE.

Mechanistic Hypothesis. Based on the structure assignment, the results from small molecule reactions, and relevant literature, we have proposed a tentative mechanistic picture for CPPE (Scheme 3). As mentioned earlier, copper acetylide 3 serves as a precatalyst by exchanging the $\mathrm{Cu}(\mathrm{I})$ with a monomer to give $\mathbf{A}$, which leads to activated monomer $\mathbf{B}$; on the other hand, it initiates the polymerization by reacting with $\mathbf{B}$ to afford an allenyl copper species $\mathbf{E}$, which might also exist in its isomeric form as a propargyl copper species.

Chain-growth from $\mathbf{E}$ could involve sequential regioselective $\gamma-\gamma / \alpha-\alpha$ couplings with two molecules of $\mathbf{B}$, which regenerates an allenyl copper species $\mathbf{G}$. Each coupling would expel a copper(I) species that re-enters the monomer activation process. Therefore, the stronger nucleophilicities of chaincarrying organocopper(I) species $\mathbf{H}$ and $\mathbf{I}$ compared to that of $\mathbf{A}$ sets the foundation of the chaingrowth nature of CPPE. The origin of the high regioselectivity during the propagation remains unclear at this point, nevertheless, it is in line with the results from analogous model reactions 
(Scheme 1). In addition, it should be pointed out that multi-nuclear copper(I) species might be

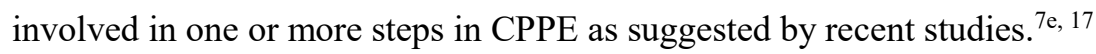

The observed end-capping structures, namely a benzyl-substituted diyne or a terminal alkyne, could be derived from the protonation of $\mathbf{H}$ or $\mathbf{I}$, respectively, the ratio of which might be determined by their relative rates in reacting with B. Finally, a minor part of the polymerization could also be initiated from a self-condensation adduct $\mathbf{J}$ and follow the same propagation mechanism. ${ }^{18}$

\section{Scheme 3. A Possible Mechanism for Cu-Catalyzed CPPE.}

- Catalyst Bifurcation

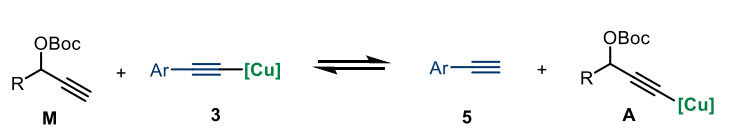

- Initiation and Propagation
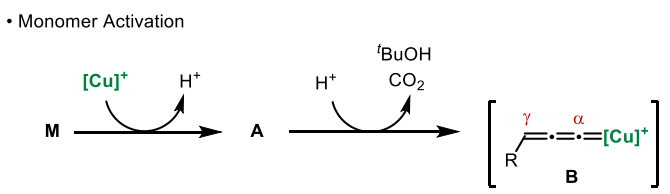

$\longrightarrow$<smiles>[R]C=C(C#CC)C(=O)O</smiles>

E

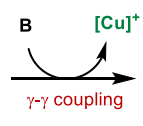

${ }_{\gamma-\gamma \text { coupling }} \mathrm{Ar} \equiv \equiv \overbrace{\mathrm{R}}^{\gamma} \equiv[\mathrm{Cu}]$

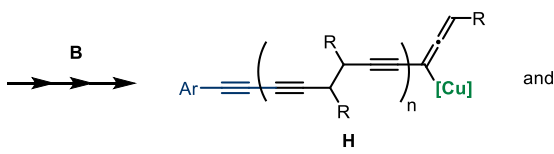

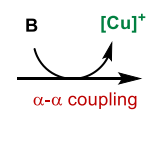
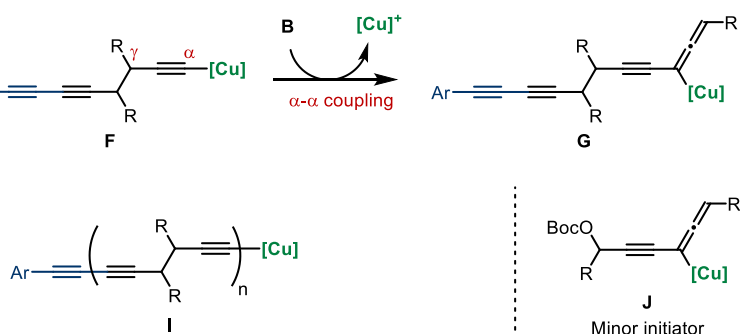

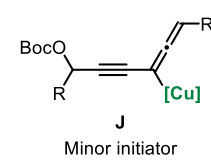

\section{Conclusion}

We have developed a new chain-growth condensation polymerization of propargyl electrophiles enabled by bifurcative copper catalysis, which leverages the reactivity difference among organocopper(I) species. Polydiynes were synthesized with good functional group tolerance and controllable MW/end groups, through the utilization of an easy-to-handle $\mathrm{Cu}(\mathrm{I})$ arylacetylide initiator/precatalyst and a phosphine ligand. The "living" character of this polymerization was 
demonstrated by the synthesis of a block copolymer. This method provides a complementary controlled access to important functional acetylenic polymers that have been traditionally obtained by step-growth methods. Mechanistic details regarding the initiation, propagation, and ligand effect are currently under investigation. Taking advantage of the chain-growth nature, the versatility of the chain-carrying organocopper(I) species, and the rich post-polymerization chemistry based on the reactive 1,3-diynes, we expect that further development of this emerging polymerization would deliver novel materials with tailored architectures and properties. 


\section{References}

1. Grubbs, R. B.; Grubbs, R. H. 50th Anniversary Perspective: Living PolymerizationEmphasizing the Molecule in Macromolecules. Macromolecules 2017, 50, 6979-6997.

2. (a) Yokozawa, T.; Ohta, Y. Transformation of Step-Growth Polymerization into Living ChainGrowth Polymerization. Chem. Rev. 2016, 116, 1950-1968. (b) Yokozawa, T.; Ohta, Y. Scope of controlled synthesis via chain-growth condensation polymerization: from aromatic polyamides to pi-conjugated polymers. Chem. Commun. 2013, 49, 8281-8310. (c) Yokozawa, T.; Yokoyama, A. Chain-growth condensation polymerization for the synthesis of well-defined condensation polymers and $\pi$-conjugated polymers. Chem. Rev. 2009, 109, 5595-5619.

3. (a) Yokozawa, T.; Asai, T.; Sugi, R.; Ishigooka, S.; Hiraoka, S. Chain-Growth Polycondensation for Nonbiological Polyamides of Defined Architecture. J. Am. Chem. Soc. 2000, 122, 8313-8314. (b) Yokoyama, A.; Iwashita, K.-i.; Hirabayashi, K.; Aiyama, K.; Yokozawa, T. Investigation of Aromatic Polyester Synthesis by the Chain-Growth Polycondensation Method. Macromolecules 2003, 36, 4328-4336. (c) Suzuki, Y.; Hiraoka, S.; Yokoyama, A.; Yokozawa, T., Chain-Growth Polycondensation for Aromatic Polyethers with Low Polydispersities: Living Polymerization Nature in Polycondensation. Macromolecules 2003, 36, 4756-4765. (d) Yokozawa, T.; Suzuki, H. Condensative Chain Polymerization in Solid-Liquid Phase. Synthesis of Polyesters with a Defined Molecular Weight and a Narrow Molecular Weight Distribution by Polycondensation. J. Am. Chem. Soc. 1999, 121, 11573-11574.

4. (a) Yokoyama, A.; Miyakoshi, R.; Yokozawa, T. Chain-Growth Polymerization for Poly(3hexylthiophene) with a Defined Molecular Weight and a Low Polydispersity. Macromolecules 2004, 37, 1169-1171. (b) Iovu, M. C.; Sheina, E. E.; Gil, R. R.; McCullough, R. D. Experimental Evidence 
for the Quasi-"Living" Nature of the Grignard Metathesis Method for the Synthesis of Regioregular Poly(3-alkylthiophenes). Macromolecules 2005, 38, 8649-8656. (c) Miyakoshi, R.; Yokoyama, A.; Yokozawa, T. Catalyst-transfer polycondensation. Mechanism of Ni-catalyzed chain-growth polymerization leading to well-defined poly(3-hexylthiophene). J. Am. Chem. Soc. 2005, 127, 17542-17547. (d) Bryan, Z. J.; McNeil, A. J. Conjugated Polymer Synthesis via Catalyst-Transfer Polycondensation (CTP): Mechanism, Scope, and Applications. Macromolecules 2013, 46, 83958405. (e) Leone, A. K.; Mueller, E. A.; McNeil, A. J. The History of Palladium-Catalyzed CrossCouplings Should Inspire the Future of Catalyst-Transfer Polymerization. J. Am. Chem. Soc. 2018, 140, 15126-15139. (f) Kang, S.; Ono, R. J.; Bielawski, C. W. Controlled Catalyst Transfer Polycondensation and Surface-initiated Polymerization of a $p$-Phenyleneethynylene-Based Monomer. J. Am. Chem. Soc. 2013, 135, 4984-4987. (g) Seo, K. B.; Lee, I. H.; Lee, J.; Choi, I.; Choi, T. L. A Rational Design of Highly Controlled Suzuki-Miyaura Catalyst-Transfer Polycondensation for Precision Synthesis of Polythiophenes and Their Block Copolymers: Marriage of Palladacycle Precatalysts with MIDA-Boronates. J. Am. Chem. Soc. 2018, 140, 4335-4343. For related catalyst transfer in polycycloaddition: (h) Sugiyama, Y. K.; Kato, R.; Sakurada, T.; Okamoto, S. Chain-Growth Cycloaddition Polymerization via a Catalytic Alkyne $[2+2+2]$ Cyclotrimerization Reaction and Its Application to One-Shot Spontaneous Block Copolymerization. J. Am. Chem. Soc. 2011, 133, 9712-9715. (i) Ravetz, B. D.; Ruhl, K. E.; Rovis, T. External Regulation of Cobalt-Catalyzed Cycloaddition Polymerization with Visible Light. ACS Catal. 2018, 8, 5323-5327.

5. (a) Sanji, T.; Iyoda, T. Transition-Metal-Free Controlled Polymerization of 2-Perfluoroaryl-5trimethylsilylthiophenes. J. Am. Chem. Soc. 2014, 136, 10238-10241. (b) Murarka, S.; Studer, A. 
Radical/Anionic S(RN)1-Type Polymerization for Preparation of Oligoarenes. Angew. Chem. Int. Ed. 2012, 51, 12362-12366. (c) Woods, E. F.; Berl, A. J.; Kalow, J. A. Photocontrolled Synthesis of n-Type Conjugated Polymers. Angew. Chem. Int. Ed. 2020, 59, 6062-6067. (d) Bonillo, B.; Swager, T. M. Chain-Growth Polymerization of 2-Chlorothiophenes Promoted by Lewis Acids. J. Am. Chem. Soc. 2012, 134, 18916-18919.

6. (a) Zhou, X.-L.; Yang, F.; Sun, H.-L.; Yin, Y.-N.; Ye, W.-T.; Zhu, R. Cobalt-Catalyzed Intermolecular Hydrofunctionalization of Alkenes: Evidence for a Bimetallic Pathway. J. Am. Chem. Soc. 2019, 141, 7250-7255. (b) Sun, H.-L.; Yang, F.; Ye, W.-T.; Wang, J.-J.; Zhu, R. Dual Cobalt and Photoredox Catalysis Enabled Intermolecular Oxidative Hydrofunctionalization. ACS Catal. 2020, 10, 4983-4989. (c) Li, R.-Z.; Liu, D.-Q.; Niu, D. Asymmetric O-propargylation of secondary aliphatic alcohols. Nature Catal. 2020, 3, 672-680. (d) Li, R. Z.; Tang, H.; Yang, K. R.; Wan, L. Q.; Zhang, X.; Liu, J.; Fu, Z.; Niu, D. Enantioselective Propargylation of Polyols and Desymmetrization of meso 1,2-Diols by Copper/Borinic Acid Dual Catalysis. Angew. Chem. Int. Ed. 2017, 56, 72137217.

7. (a) Imada, Y.; Yuasa, M.; Nakamura, I.; Murahashi, S.-I. Copper(I)-Catalyzed Amination of Propargyl Esters. Selective Synthesis of Propargylamines, 1-Alken-3-ylamines, and (Z)Allylamines. J. Org. Chem. 1994, 59, 2282-2284. (b) Detz, R. J.; Delville, M. M.; Hiemstra, H.; van Maarseveen, J. H. Enantioselective copper-catalyzed propargylic amination. Angew. Chem. Int. Ed. 2008, 47, 3777-3780. (c) Hattori, G.; Matsuzawa, H.; Miyake, Y.; Nishibayashi, Y. Copper-catalyzed asymmetric propargylic substitution reactions of propargylic acetates with amines. Angew. Chem. Int. Ed. 2008, 47, 3781-3783. (d) Nishibayashi, Y. Transition-Metal-Catalyzed Enantioselective Propargylic Substitution Reactions of Propargylic Alcohol Derivatives with Nucleophiles. Synthesis 
2012, 44, 489-503. (e) Nakajima, K.; Shibata, M.; Nishibayashi, Y. Copper-Catalyzed Enantioselective Propargylic Etherification of Propargylic Esters with Alcohols. J. Am. Chem. Soc. 2015, 137, 2472-2475. (f) Zhang, D.-Y.; Hu, X.-P. Recent Advances in Copper-Catalyzed Propargylic Substitution. Tetrahedron Lett. 2015, 56, 283-295.

8. (a) Sakata, K.; Nishibayashi, Y. Mechanism and Reactivity of Catalytic Propargylic Substitution Reactions via Metal-Allenylidene Intermediates: A Theoretical Perspective. Catal. Sci. Technol. 2018, 8, 12-25. (b) Díez-González, S. Copper(I)-Acetylides: Access, Structure, and Relevance in Catalysis. Adv. Organomet. Chem. 2016, 66, 93-141. (c) Wang, B. C.; Wang, Y. N.; Zhang, M. M.; Xiao, W. J.; Lu, L. Q. Copper-Catalyzed Decarboxylative Cyclization via Tandem C-P and C-N Bond Formation: Access to 2-Phosphorylmethyl Indoles. Chem. Commun. 2018, 54, 3154-3157.

9. (a) Liu, J.; Lam, J. W.; Tang, B. Z. Acetylenic Polymers: Syntheses, Structures, and Functions. Chem. Rev. 2009, 109, 5799-5867. (b) Hu, R.; Lam, J. W. Y.; Tang, B. Z. Recent Progress in the Development of New Acetylenic Polymers. Macromol. Chem. Phys. 2013, 214, 175-187.

10. Tedeschi, C.; Saccavini, C.; Maurette, L.; Soleilhavoup, M.; Chauvin, R. 1,4-Diynes from alkynyl-propargyl coupling reactions. J. Organomet. Chem. 2003, 670, 151-169.

11. The quantitation of $2^{\prime}$ was prevented by overlapping signals in ${ }^{1} \mathrm{H}$ NMR of the crude mixture. Therefore an estimation of cross-condensation $>20 \%$ was made based on 2 .

12. Huang, X.; Anderson, K. W.; Zim, D.; Jiang, L.; Klapars, A.; Buchwald, S. L. Expanding PdCatalyzed C-N Bond-Forming Processes: The First Amidation of Aryl Sulfonates, Aqueous Amination, and Complementarity with Cu-Catalyzed Reactions. J. Am. Chem. Soc. 2003, 125, 6653-6655.

13. Shi, W.; Lei, A. 1,3-Diyne Chemistry: Synthesis and Derivations. Tetrahedron Lett. 2014, 55, 
2763-2772.

14. Evano, G.; Jouvin, K.; Theunissen, C.; Guissart, C.; Laouiti, A.; Tresse, C.; Heimburger, J.;

Bouhoute, Y.; Veillard, R.; Lecomte, M.; Nitelet, A.; Schweizer, S.; Blanchard, N.; Alayrac, C.;

Gaumont, A. C. Turning Unreactive Copper Acetylides into Remarkably Powerful and Mild Alkyne

Transfer Reagents by Oxidative Umpolung. Chem. Commun. 2014, 50, 10008-10018.

15. Häussler, M.; Zheng, R.; Lam, J. W. Y.; Tong, H.; Dong, H.; Tang, B. Z. Hyperbranched Polyynes:

Syntheses, Photoluminescence, Light Refraction, Thermal Curing, Metal Complexation, Pyrolytic

Ceramization, and Soft Magnetization. J. Phys. Chem. B 2004, 108, 10645-10650.

16. Zhang, H.; Zheng, X.; Xie, N.; He, Z.; Liu, J.; Leung, N. L. C.; Niu, Y.; Huang, X.; Wong, K.

S.; Kwok, R. T. K.; Sung, H. H. Y.; Williams, I. D.; Qin, A.; Lam, J. W. Y.; Tang, B. Z. Why Do

Simple Molecules with "Isolated" Phenyl Rings Emit Visible Light? J. Am. Chem. Soc. 2017, 139, 16264-16272.

17. Worrell, B. T.; Malik, J. A.; Fokin, V. V. Direct Evidence of a Dinuclear Copper Intermediate in Cu(I)-Catalyzed Azide-Alkyne Cycloadditions. Science 2013, 340, 457-60.

18. An alternative mechanism involving an in situ formed $\mathrm{Cu}$-complexed [5]cumulene species as the monomer is depicted in Figure S19. This hypothesis cannot be ruled out at this point but is less favored due to the instability of high cumulenes. 


\section{Data availability}

The authors declare that all other data supporting the findings of this study are available within the article and Supplementary Information files, and also are available from the corresponding author upon reasonable request.

\section{Acknowledgements}

Financial support was provided by the Natural Science Foundation of China (21901011), and startup fund from the College of Chemistry and Molecular Engineering, Peking University and BNLMS. The authors would like to thank Prof. Zichen Li and Prof. Jian Pei (PKU) for insightful discussions, and Prof. Hua Lu and Dr. Wei Xiong (PKU) for assistance with MALDI-TOF mass spectroscopy.

\section{Author contributions}

R. Z. conceived of the idea, guided the project and wrote the manuscript, with feedbacks from the other authors. H.-L. S., D.-Q. L., J.-J. W. and R. Z. performed the experiments. H.-L. S., D.-Q. L., J.-J. W., D. N., and R. Z. analyzed the results. 